\title{
Blood Biomarkers for Alzheimer's Disease: Much Promise, Cautious Progress
}

Ashvini Keshavan,

Amanda Heslegrave, 2

Henrik Zetterberg, 2,3,4

Jonathan M. Schott, 1 ,

Phone 00442034483856

Emailj.schott@ucl.ac.uk

Dementia Research Centre, UCL Institute of Neurology, National Hospital for Neurology and Neurosurgery, Box 16, Queen Square, London, WC1N $3 \mathrm{BG}$ UK

2 Department of Molecular Neuroscience, UCL Institute of Neurology, London, UK

3 Department of Psychiatry and Neurochemistry, Institute of Neuroscience and Physiology, The Sahlgrenska Academy at University of Gothenburg, Sahlgrenska University Hospital, Mölndal, Sweden

4 Clinical Neurochemistry Laboratory, Sahlgrenska University Hospital, Mölndal, Sweden

\section{Abstract}

Biomarkers in Alzheimer's disease (AD) have the potential to allow early and more accurate diagnosis, predict disease progression, stratify individuals and track response to candidate therapies in drug trials. The first fluid biomarkers reflecting aspects of $\mathrm{AD}$ neuropathology were identified in cerebrospinal fluid (CSF) in the 1990s. Three CSF biomarkers (amyloid- $\beta$ 1-42, total tau and phospho-tau) have consistently been shown to have diagnostic utility and 
are incorporated into the new diagnostic criteria for AD. These markers have also been shown in longitudinal studies to predict conversion of mild cognitive impairment to AD. However, a key issue with the use of CSF biomarkers as a screening test is the invasiveness of lumbar puncture. Over the last 20 years there has been an active quest for blood biomarkers, which could be easily acquired and tested repeatedly throughout the disease course. One approach to identifying such markers is to attempt to measure candidates that have already been identified in CSF. Until recently, this approach has been limited by assay sensitivity, but newer platforms now allow single molecule-level detection. Another approach is identification of candidates in large multiplex panels that allow for multiple analytes to be quantified in parallel. While both approaches show promise, to date no blood-based biomarker or combination of biomarkers has sufficient predictive value to have utility in clinical practice. In this review, an overview of promising blood protein candidates is provided, and the challenges of validating and converting these into practicable tests are discussed.

\section{AQ1}

H. Zetterberg and J. M. Schott are joint senior author of this article.

\section{Key Points}

Many studies have identified candidates for blood biomarkers of Alzheimer's disease (AD) but replication has been problematic.

The two main candidates showing promise currently are plasma total tau and serum neurofilament light chain.

New techniques such as multiplexing and use of more sensitive assays are likely to expand and improve blood biomarker research.

\section{Introduction}

The neuropathological signature of Alzheimer's disease (AD) is neuronal loss with deposition of amyloid- $\beta(A \beta)$ in extracellular plaques and accumulation of hyper-phosphorylated tau protein in intracellular neurofibrillary tangles.

Extrapolation from post-mortem studies suggests that in most cases these proteins propagate through the brain in a reliable progression, which is reflected in neuropathological staging systems for AD [1]. These studies showed that early stage AD neuropathology exists in individuals who are asymptomatic, 
paving the way for pre-symptomatic diagnosis and clinical trials aiming to prevent cognitive decline. Given the obvious limitations of obtaining brain tissue during life, there has been considerable interest in discovering diseasespecific biomarkers both in the symptomatic and pre-symptomatic phases.

Ideal biomarkers have different characteristics based on the information they aim to give [1]. For example, an ideal diagnostic biomarker would reliably reflect in vivo pathology with high sensitivity and specificity. A screening biomarker would combine at least moderate sensitivity with high specificity and low cost. Conversely, a marker of progression may be downstream of the initial pathology but reliably track change over time. For all biomarkers, reliability, cost, and ease of acquisition and processing are important considerations.

The currently available biomarkers for AD include structural imaging (e.g. magnetic resonance imaging [MRI] and computed tomography), functional imaging (e.g. 18-fluorodeoxyglucose positron emission tomography [PET]), molecular imaging (e.g. A $\beta$ and tau PET) and fluid biomarkers (from cerebrospinal fluid [CSF], blood and urine). The focus of this review is fluid biomarkers in $\mathrm{AD}$.

\section{Cerebrospinal Fluid Biomarkers: Established and Emerging}

Studies have shown that low CSF A 342 [2], high CSF total tau (t-tau) [3] and high CSF phospho-tau [4] in life are correlated with a clinical diagnosis of AD, and also with severity of AD pathology post-mortem $[5,6]$. Moreover, several large multicentre studies have shown this pattern to predict which patients with mild cognitive impairment (MCI) will progress to $\mathrm{AD}[7,8,9]$. This implies that the change in CSF biomarkers must occur during the preclinical stage of AD. This CSF biomarker signature is now established in both the International Working Group (IWG)-2 and National Institute on Aging (NIA) diagnostic criteria for $\mathrm{AD}$, in pre-symptomatic, prodromal (MCI), typical and atypical forms of $\mathrm{AD}[3,10,11,12,13]$.

Newer CSF biomarkers that reflect other aspects of AD remain an active topic of research. The post-synaptic degeneration biomarker neurogranin has been identified in multiple cohorts as being able to differentiate AD from control with an effect size (defined as fold change in the mean biomarker concentration between $\mathrm{AD}$ and control groups) of 1.9 in a recent meta-analysis by Olsson et 
al. [14], and to differentiate MCI patients who will progress to AD [effect size 1.5] [15]. Other studies have provided evidence that numerous other CSF markers may be able to distinguish AD from controls, including heart fatty acid binding protein [16], neuron-specific enolase [17], neurofilament light chain (NFL) [18], YKL-40 [19] and visinin-like protein-1 [20], with effect sizes ranging from 1.3 to 2.3 . However, none is currently more diagnostically useful than the established markers $A \beta 42$ and tau, suggesting that the true utility of these additional markers will come from understanding their relationship to the underlying pathology, and in using them to ask more refined questions. For example, a diagnostic marker is most useful when it can differentiate AD from other dementias. This has been recently observed by Wellington et al. [21] for neurogranin, which rises specifically in AD but not in other diseases such as Lewy body dementia (LBD) and behavioural variant frontotemporal dementia (FTD) which often are in the differential diagnosis of AD. Another more specific function of a diagnostic biomarker would be to differentiate subtypes of AD. Paterson et al. [22] have shown that the latter may be the case for CSF NFL, which is higher in the CSF of patients in the frontal variant subgroup of $\mathrm{AD}$ than in other 'atypical' variants of $\mathrm{AD}$ as defined by the IWG-2 criteria; this represents a group that tends to have younger onset and more aggressive disease.

\section{AQ2}

'Traditional' assay development has been on a single candidate molecule approach, devising specific enzyme-linked immunosorbent assays (ELISAs) for proteins thought to be relevant to the disease. This approach has its limitations: it is slow and relies on a priori assumptions of the role of the candidate analyte in disease pathogenesis. Thus, many groups have turned to employing multiplexing approaches in CSF $[23,24]$. A recent example is Heywood et al.'s mass spectrometry-based targeted proteomics assay in CSF from two independent cohorts[63], I have been unable to assign the correct numbering to this reference, which was inserted twice (once fully and once partially) as reference 63 and 64 . Please reassign it as number 25 , adjusting the numbering of the further references below, including those in the table. AK. Which demonstrated 23 proteins (six of which were novel) that differentiate AD and LBD from controls. Four proteins (two of which were novel) differentiated AD from both LBD and controls. Many of these markers did not show a relationship with CSF A $\beta 42$, ttau or phospho-tau, implying that their association with the pathology of $\mathrm{AD}$ may be unrelated to amyloid plaques and tau neurofibrillary tangles. 


\section{Minimally Invasive Tests}

While CSF biomarkers have proven utility, obtaining CSF remains a relatively invasive procedure. Lumbar puncture is not without complications (the commonest of which is headache) and it is relatively contraindicated in patients with clotting disorders and those who are taking anticoagulant medication. It requires a skilled operator and appropriate facilities to take, process and store samples, which are sensitive to handling errors. This set of systems and processes adds to the overall cost of the test. The optimal biomarker(s) would therefore be obtained via a non-invasive, relatively cheap and easily repeatable test. Blood, which is collected paired with CSF in most major fluid biomarker research initiatives, provides this opportunity.

\section{Blood Biomarkers}

\subsection{The Candidate Approach}

Identification of peripheral biomarkers reflecting central nervous system (CNS) dysfunction is challenging for many reasons. First, molecules from the brain must cross the blood-brain barrier. Second, their concentration in blood is likely to be much smaller than that in CSF, due to the much higher volume of dilution in blood. Third, the high concentration of plasma proteins can be either a sink for secreted proteins from the brain (due to binding or enzymatic breakdown) or a source of similar or identical proteins. Taking the example of plasma $A \beta 42$, many of these factors come into play. A $\beta 42$ is present at very low concentrations in plasma, it is prone to plasma protein binding and may be eliminated by enzyme activity. These factors may explain why plasma A $\beta 42$ does not correlate well with the CSF profile [25, 26, 27, 28] and there are mixed reports of inverse correlation [29] and of lack of correlation [30] of plasma A $\beta 42$ with amyloid PET. Further problems with plasma $A \beta 42$ are its susceptibility to technical factors such as aliquot volume and delay to freezing samples [31].

To date, only one blood biomarker has been shown to distinguish AD from control subjects across multiple patient populations. Plasma t-tau was shown in a recent meta-analysis across six comparisons [32,33] to have an effect size of 1.95 (95 \% CI 1.12-3.38; $n=271$ AD, 394 controls). Yet even within this metaanalysis there was significant variation depending on the method used to quantify t-tau. One of these studies showed a significant inverse effect [34] and 
one showed no appreciable effect [35] — both used conventional ELISA methods that were not optimised to measure the low concentrations of tau in the blood. A more sensitive immunomagnetic reduction method was used in three other comparisons derived from two studies by the same group [36,37] and showed effect sizes consistent with the results of the overall meta-analysis, but these results, using this particular measurement technique, are in need of independent replication. The final study [38] utilised a single molecular digital array platform that is able to quantify $\mathrm{t}$-tau at the single molecule level and proved to have the highest sensitivity of the three methods [39]. This study compared AD with $\mathrm{MCI}$ and control subjects from a Swedish cohort, showing that while plasma $\mathrm{t}$-tau differentiates $\mathrm{AD}$ from control, there is no significant effect for MCI versus control. The former finding has been replicated in the Alzheimer's Disease Neuroimaging Initiative (ADNI) cohort and the Swedish BioFINDER study [40] and the latter finding has been independently replicated in a larger cohort from the Mayo Clinic Study of Aging [41], using the same platform. While the difference in plasma t-tau levels between MCI and control did not reach statistical significance, there was a significant inverse correlation between plasma tau and cognitive performance (in the global, memory and attention/executive function domains), as well as between plasma t-tau and cortical thickness in an AD region of interest defined on MRI. A summary of the studies published thus far is provided in Table 1 .

\section{Table 1}

Key blood biomarker studies in Alzheimer's disease showing significant results using the candidate approach

\begin{tabular}{|c|c|c|c|}
\hline $\begin{array}{l}\text { Study, } \\
\text { year }\end{array}$ & Assay & $\begin{array}{l}\text { Cohort } \\
(n)\end{array}$ & $\begin{array}{l}\text { Summary of results (effect } \\
\text { size } 95 \% \text { CI) }\end{array}$ \\
\hline
\end{tabular}

Total plasma tau

Sparks et al. 2012

[34]

ELISA

[34]

Chiu et

al. 2013

[36]

Immunomagnetic

reduction

Chiu et

al. 2014

[37]
Immunomagnetic

reduction
AD (49)

Control

(110)

$2 \mathrm{AD}$

groups

$(31+30)$

Control

(107)

Zetterberg Single molecular digital

$\mathrm{AD}(10)$

Control

(30)
Tau lower in AD (effect size $0.6-0.7$ )
Tau higher in AD (effect size 2.3-9)

d

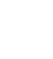


et al. 2013 immunoarray

Control

$1.3-3)$

[38 ]

(25)

Dage et

al. 2016

[41]
Single molecular digital immunoarray
Tau in MCI was not statistically significantly higher than in controls but

MCSA

cohort:

MCI

(161)

Control

(378) after adjustment for age, sex, education and APOE genotype, higher tau was associated with:

1. Worse performance in tests of global cognition, memory and attention; and 2. Reduced cortical thickness in an AD signature region on MRI

NFL, serum

Bacioglu et al. 2016 [47]
ELISA adapted to electrochemiluminescent platform
Control

(35)

MCI

(33)

AD (34)

IPD (32)

DLB

(20)

MSA

(17)

PSP (24)

CBS

(10)
Serum NFL higher in AD (effect size $\sim 3$ ) but not statistically significant for MCI

Significant correlation between cognitive performance on MMSE and CSF NFL or serum NFL in $\mathrm{AD}$

$A D$ Alzheimer's disease, $C B S$ corticobasal syndrome, $C I$ confidence interval, $C S F$ cerebrospinal fluid, $D L B$ dementia with Lewy bodies, ELISA enzyme-linked immunosorbent assay, IPD idiopathic Parkinson's disease, $M C I$ mild cognitive impairment, MCSA Mayo Clinic Study of Aging, MMSE Mini-Mental State Examination, $M R I$ magnetic resonance imaging, $M S A$ multisystem atrophy, $N F L$ neurofilament light chain, $P S P$ progressive supranuclear palsy

\section{AQ4}

\section{AQ5}

While plasma tau shows some promise, interpretation of the various studies is not easy, given that plasma t-tau is known to vary widely even in healthy individuals; and tau elevation is not specific to $\mathrm{AD}$, as it is seen in many diseases with rapid neuronal destruction (e.g. prion disease) as well as after traumatic brain injury [42]. There is also a lack of correlation between CSF and plasma t-tau within individuals [35]. Further studies in different cohorts are needed to understand what plasma tau elevation means before it can be more routinely used in research and certainly before any potential clinical translation. 
relationship between serum NFL and the progression of neurodegenerative diseases. NFL in CSF may discriminate FTD from other types of dementia [43] and be an indicator of disease severity in FTD [44]. More recently, there have been reports of serum NFL also reflecting disease severity in FTD $[45,46]$ and correlating with the annualised frontal lobe atrophy rate in FTD [46]. In an extensive body of work spanning mouse APP-PS1 (amyloid precursor proteinpresenilin 1) mutant models and human CSF and blood sampling, Bacioglu et al. [47] showed that both CSF and serum NFL are raised in AD compared with control patients. Serum NFL was highest in patients with tauopathies (with effect sizes $\sim 3$ to 6 ) and moderately high in those with $\mathrm{AD}$ and atypical parkinsonian syndromes (with effect sizes $\sim 3$ ), but was no different in those with idiopathic Parkinson's disease compared with controls, lending support to the view that this may be a useful test in distinguishing idiopathic Parkinson's disease from atypical parkinsonism. Serum NFL broadly correlated with CSF within individuals (a finding that has been corroborated by others for both serum [48] and plasma [49]) and also correlated with Mini-Mental State Examination scores. Treatment of the mouse APP-PS1 models with a $\beta$ secretase-1 inhibitor, which reduces the generation of $A \beta 42$ and the formation of amyloid plaques, led to a reduction in both CSF and plasma NFL, which was not observed in the untreated APP-PS1 mice. Taken together, these findings raise the prospect of using blood NFL as a possible treatment response biomarker in AD trials, as, unlike plasma t-tau, serum NFL seems to track the CSF levels and the progression of disease. Notably, a statistically significant elevation in serum NFL was not seen in MCI, but this may yet change as serum NFL is measured in pre-symptomatic and mildly symptomatic subjects (such as in the Dominantly Inherited Alzheimer's Network cohort) with higher sensitivity assays such as single molecular array. It will be interesting in such cohorts to determine whether models that combine serum NFL levels with serial MRI data could provide more predictive value for disease severity than using either test alone.

\section{AQ6}

Any such efforts will need to adopt a standardised approach across centres to allow for cross-validation, and there is now an international working group preanalytic processing guideline [50], which will continue to evolve as additional research findings become available. The guideline refers to "controllable" and "uncontrollable" variables. These match technical variables (in blood collection/handling/storage) and patient factors (such as demographics, genotype, co-morbidities and lifestyle factors), respectively. While the former 
should be standardised, collecting information on the latter will allow for stratification and interesting between-group comparisons that may ultimately inform the application of a particular biomarker as a screening, diagnostic, prognostic or therapeutic indicator.

\section{AQ7}

\subsection{Multiplexing}

As has occurred in the CSF field, multiplexing approaches to plasma proteomics have been employed by several investigators in an effort to define groups of proteins that may inform pathways to the development and progression of AD. A selection of some notable studies is provided in Table 2. The earliest of these studies, by Ray et al. [51] in 2007, used a sandwich ELISA immunoassay platform and identified an 18-analyte panel of proteins that segregated AD from controls. However, in an attempt at replication in another cohort (ADNI), by using seven of the analytes that gave a combined diagnostic accuracy of $90 \%$ in the Ray et al. [51] study, $61 \%$ diagnostic accuracy was achieved, and the incorporation of a different 89-analyte panel increased this to $70 \%$ [52]. These results, however, have been difficult to replicate [53,54]. Doecke et al. [55] cross-validated panels of biomarkers obtained from AIBL (Australian Imaging, Biomarkers and Lifestyle study) against ADNI, and found just two biomarkers that had individual effect sizes greater than 1.5 that were common to both cohorts: insulin-like growth factor binding protein 2 and pancreatic polypeptide. However, when a multivariate model using a panel of eight plasma biomarkers was added to the predictive capacity of a model based on age, sex and $A P O E$ genotype, the biomarker panel was only able to increase sensitivity and specificity from 77 to $83 \%$. This emphasises the importance of assessing how much actual additional predictive information is provided by these tests, as it ultimately relates to the cost-benefit analyses that will determine their ability to translate into more general research or clinical settings.

\section{Table 2}

Key blood biomarker studies in Alzheimer's disease showing significant results multiplexing approach. This represents a non-exhaustive list of relevant studies Study,
year
Cohort (n)

AD (41)

Control (30)
Summary results 
Study also

included

sera from

Zhang et $\mathrm{LC}+\mathrm{SDS}-\mathrm{PAGE}+\mathrm{MS}$

al. 2004

[56]
Cross-validation with WB and ELISA patients

with

insulin-

resistant

diabetes

mellitus and

congestive

heart failure

patients
In serum: 12

proteins

elevated in

$\mathrm{AD} ; 1$ protein reduced in AD

histidine-ri

glycoprote specific els in $\mathrm{AD}$ but other disea 1 protein $\mathrm{s}$ specific rer in AD: $\alpha-1$ glycoprote No statem diagnostic sensitivity specificity

Sensitivity and specifi $96 \%$ achie the 2 mode contributio individual not analyse

MCI (33)

al. 2005

$\mathrm{AC}+\mathrm{MS}$

Control

(207)

In serum: 2

models

generated by

peptide

spectral

signatures

$\mathrm{AD}(85)$

MCI (47)

Other

dementia

(11)

Other

Ray et al.

2007

[51]

Cytokine antibody

microarray; sandwich

ELISA

neurological

disease (22)

Rheumatoid

arthritis

(16)

Control (79)

In plasma:

120 cell signalling proteins probed; 18 proteins identified
$91 \%$ accu predicting converting $100 \%$ accl predicting converting $\mathrm{AD}$ demen
Doecke et al. 2012

[55]
Soares et al. 2009 [52]
Sandwich ELISA
ADNI

cohort: AD

(61)

Control

(952)
In serum: 151 protein panel, including 8 proteins from Ray et al. [51]
$61 \%$ accu classifying control; un replicate $\mathrm{r} \epsilon$ Ray et al. [

IGFBP2 ar each had ir effect sizes these and s other mark been replic other studi The 8 mos predictive which wer by each of statistical 1 were able 1 increase th sensitivity specificity predictive based on a 
education :

APOE gen

from 77 to

Penn

cohort:

Control

(126)

MCI (16)

$\mathrm{AD}(88)$

Other

dementias

(37)

WU cohort:

Control

(242)

CDR 0.5 or

Multiplexed

$\mathrm{Hu}$ et al.

2012

[61] immunoassay platform for a 190 protein panel $A P O E$ genotyping
In plasma in

Penn and WU cohorts:

17 candidate proteins

identified in

univariate

analysis

across both

cohorts to be

associated

with clinical

MCI/AD; 5

replicated by

another study

in serum

6 of these

survived

multivariate

analysis and

correction for

age and sex

In fasting

plasma in

ADNI:

6 candidates

were

identified by

univariate

analysis

4 of these

showed

correlations

with disease

status defined

by CSF

2 of these

survived

multivariate
6 markers common tc testing coh

$\mathrm{MCI} / \mathrm{mild}$ dementia/t antitrypsin CRP, N-ter pro B-type natriuretic osteoponti1 serum amy Individual sizes range 0.8 to 1.1 . In the ADI validation when disec was define CSF signa1 CSF A $\beta 42$ levels $<19$. and a CSF 42 ratio $>0$ markers $\mathrm{w}$ identified: BNP, CRP, pancreatic polypeptid latter 3 we independe1 cholinester inhibitor $\mathrm{u}$ BNP and $p$ polypeptid significant correlation $A P O E 4$ all number an CSF A $\beta 42$ pancreatic polypeptid showed sig linear corri with the $C$ : tau/A $\beta 42$ APOE, BN CRP were common tc cohorts.

The main limitations study inclu 1. methode differences the three $\mathrm{c}$ the sample 
analysis and correction for age and sex

collection

vs. non-fas

2. the grou

$\mathrm{MCI} / \mathrm{mild}$

dementia/t controls in analysis, $\mathrm{r} \epsilon$ in an inabi comment $\mathrm{c}$ correlation these mark disease ser 3. small in effect sizes contributio plasma bio to explaini variance in markers in ADNI cohı

3 markers

AD (142)

Control

(174)

Other dementias (88) et al. 2012

[53]
Quantitative multiplex ELISA electrochemiluminescence multiarray
Trushina et al. 2013 [57]

\section{$\mathrm{LC}+\mathrm{MS}$}

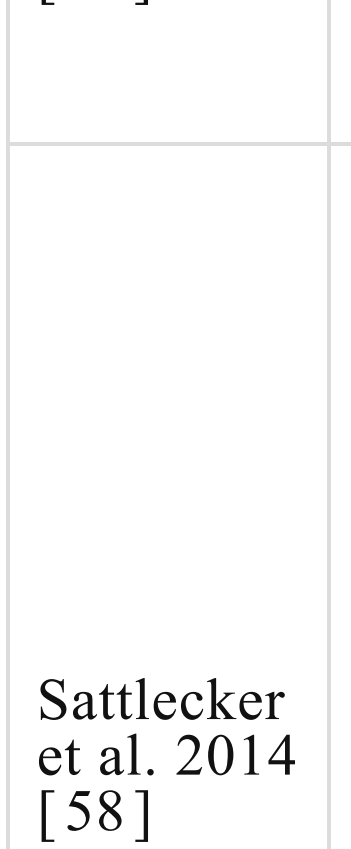

Aptamer-based microarray

\begin{tabular}{l|l|} 
AD $(331)$ & of the \\
MCI (106) & outcome \\
Control & measures; 13- \\
(211) & member \\
43 MCI & panel selected \\
patients & 20 proteins \\
converted to & associated \\
AD within a & with \\
year & conversion of \\
& $\begin{array}{l}\text { MCI to AD } \\
\text { but none } \\
\text { passed } \\
\text { multiple } \\
\text { testing } \\
\text { corrections }\end{array}$ \\
&
\end{tabular}

distinguish from contr from other dementias; diagnostic $63 \%$

23 altered pathways $i$ and 20 in ( MCI vs. cc with a fals discovery 1 $<5 \%$

Biomarker correlated different $o$ measures, including: region-spe atrophy on (e.g. pancr prohormon correlated hippocamp volume) rate of $\operatorname{cog}$ decline (e.: clusterin)

13 protein showed se1 $67 \%$, spec $64 \%$, posi predictive $70 \%$ 
screen:

Control

elderly (53)

$\mathrm{MCI} / \mathrm{mild}$

AD (53, including

18

converters

over

Mapstone

et al. 2014

$\mathrm{LC}+\mathrm{MS}$

2 years)

Targeted

panel

(separate

cohort):

Normal

elderly (20)

$\mathrm{MCI} / \mathrm{mild}$

AD (21,

including

10

converters

over

2 years)

Casanova et al. 2016 [54]
Identical to Mapstone et al. [62]
In fasting plasma: 10 plasma lipids
10 lipid pa to distingu converters normal cor with diagn precision 9 sensitivity specificity $90 \%$

Able to dis current AL controls W: precision $\mathrm{C}$

Larger-sca attempt at validating lipid panel Mapstone । [62] in ser samples co after overn BLSA cohı diagnostic for disting! MCI-to-AI converters controls w: sensitivity and specifi AGES-RS diagnostic for disting 1 MCI-to-AI converters controls w: sensitivity specificity Across bot cohorts, ab distinguish $\mathrm{AD}$ from $\mathrm{c}$ with precis 48-58\%

Other mets flagged in screen as b useful discrimina BLSA cohi 
not replica

AGES-RS

Jaeger et al. 2016 [60]
Antibody microarray
$\mathrm{AD}(47)$

Control (52) SvPPA (92)
600 secreted cell

signalling

proteins

probed

3 groups o

identified 1

discrimina

from contr

from svPP

'complem $\epsilon$

'apoptosis'

'growth

resulationr

of growth'

including $\varepsilon$

TGF $\beta / G D I$

pathway w

then invest

post-morte

brain homs

and in mur

hippocamp

culture mo

39 proteins

identified 1

over- or un

expressed

$\mathrm{AD}$ and sv

$A \beta$ amyloid- $\beta, A C$ affinity chromatography, $A D$ Alzheimer's disease, $A D N I$ Alzhein Disease Neuroimaging Initiative, $A G E S-R S$ Age, Gene/Environmental Susceptibilit: Reykjavik Study, AIBL Australian Imaging, Biomarkers and Lifestyle study, $A P O E^{-}$ apolipoprotein E, $B L S A$ Baltimore Longitudinal Study of Ageing, $B N P$ brain natriur peptide, $C D R$ Clinical Disease Rating scale as per the NINCDS-ADRDA (National of Neurological and Communicative Disorders and Stroke-Alzheimer's Disease anc Disorders Association) 1984 criteria for Alzheimer's disease, $C E A$ carcinoembryoni antigen, $C R P$ C-reactive protein, $C S F$ cerebrospinal fluid, $E G F$ epidermal growth $\mathrm{f}^{2}$ $E G F R$ epidermal growth factor receptor, ELISA enzyme-linked immunosorbent assa $I G F B P 2$ insulin-like growth factor binding protein $2, I L-17$ interleukin-17, $L C$ liqui chromatography, $M C I$ mild cognitive impairment, $M I P-1 \delta$ macrophage inflammator protein-1 $\delta, M S$ mass spectrometry, $P D G F-B B$ platelet-derived growth factor $\mathrm{BB}, P \epsilon$ University of Pennsylvania cohort, $P P Y$ pancreatic polypeptide, $S D S-P A G E$ sodium sulphate polyacrylamide gel electrophoresis, $s v P P A$ semantic variant primary progr aphasia, $T G F \beta / G D F / B M P$ transforming growth factor- $\beta /$ growth differentiation factc morphogenetic protein, $t$-tau total tau, $V C A M$ vascular cell adhesion molecule, $W B$ blot, $W U$ Washington University cohort

Other multiplexing techniques have used mass spectroscopy, preceded by either liquid chromatography or differential matrix or surface adsorption to enrich samples for proteins at low concentration. While this type of approach does not rely on the availability of specific antibodies to the analytes of interest, it is this very point that makes the data noisy. Zhang et al. [56] piloted this approach by identifying four proteins in $\mathrm{AD}$ versus control sera, using mass spectroscopy, which was then cross-validated using immunoassays (ELISA and Western blot). 
Similar methods have been employed to compare signatures of metabolites in CSF and plasma of controls, MCI and AD patients [57]. A newer method is the use of aptamer-based microarrays, which use oligonucleotides that function with high specificity, almost like 'chemical antibodies', but are more thermally stable and might therefore be more clinically translatable [58].

Multiplexing provides clear advantages by allowing large numbers of candidate biomarkers to be screened, provided that these replicate well across crosssectional and longitudinal cohorts. Although to date replication has largely been lacking, the primary 'hits' obtained so far have often segregated into classes of proteins identified from other realms of biology, for example from the 'inflammatory cascade', 'lipid metabolism', 'complement' and other groups. However, it is uncertain to what extent one can extrapolate the role of these proteins from blood to a distant underlying pathology in the CNS. Data-driven approaches also have other disadvantages. Firstly, to quote Lopez et al. [59], "Given a sufficiently large pool of potential proteins and peptides, one is almost certain to identify a pattern that discriminates between persons with and without disease within any given data set". This is the age-old problem of multivariate analysis, which can be mitigated to some extent by statistical rigour, but ultimately requires replication in independent cohorts. Secondly, biomarkers may be able to answer more powerful questions if their relationship to pathology, and indeed to factors such as healthy aging or other dementia pathologies, is known. We can begin to derive such knowledge by integrating diverse research tools such as genomics and transcriptomics with proteomics. A recent example is the work of Jaeger et al. [60] who used an in-house immune microarray to probe a library of 600 secreted signaling proteins in plasma from AD patients, semantic dementia patients and healthy controls. They identified protein clusters that map to 'complement', 'apoptosis' and 'regulation of growth', many of which were hits on previous multiplex analyses, but a novel pathway, the TGF $\beta /$ GDF/BMP (transforming growth factor- $\beta /$ growth differentiation factor/bone morphogenetic protein) cluster, was identified. The potential relevance of this pathway was supported through the demonstration of a large number of single nucleotide polymorphisms at the gene level in AD patients, and correlation with lower GDF3 protein levels in post-mortem cortical extracts from AD patients. Whilst replication in other cohorts and validation using more conventional immunoassays such as ELISA will be required, the identification of this pathway illustrates that an integrated approach may prove to be a way of discovering new biomarkers and elucidating their function, with the ultimate aim of identifying therapeutic targets. 


\section{Moving Forward}

Despite the inherent problems in developing blood-based biomarkers that accurately reflect brain biochemistry, ever more sensitive instruments and unbiased methodologies to screen large numbers of proteins concurrently mean that there is now a very real prospect of clinically useful blood-based biomarkers for AD. Blood measures of tau and of NFL are currently leading blood-based biomarker candidates; further work is required to understand what role they may play as diagnostic, prognostic or outcome measures in AD.

\section{Compliance with Ethical Standards}

Conflict of interest Ashvini Keshavan and Amanda Heslegrave have nothing to declare. Henrik Zetterberg is one of the founders of Brain Biomarker Solutions in Gothenburg AB, a GU Venture-based platform company at the University of Gothenburg, Sweden. Jonathan M. Schott has funding from Alzheimer's Research UK, the Medical Research Council, AVID Radiopharmaceuticals, the Wolfson Foundation, the Brain Research Trust and EU Horizon 20:20 but none has influenced the preparation of this paper.

Funding No funding was received for the preparation of this paper.

\section{References}

1. Dubois B, Feldman HH, Jacova C, et al. Advancing research diagnostic criteria for Alzheimer's disease: the IWG-2 criteria. Lancet Neurol. 2014;13(6):614-29.

2. Motter R, Vigo-Pelfrey C, Kholodenko D, et al. Reduction of betaamyloid peptide42 in the cerebrospinal fluid of patients with Alzheimer's disease. Ann Neurol. 1995;38:643-8.

3. Vandermeeren M, Mercken M, Vanmechelen E, et al. Detection of tau proteins in normal and Alzheimer's disease cerebrospinal fluid with a sensitive sandwich enzyme-linked immunosorbent assay. J Neurochem. 1993;61:1828-34. 
4. Vanmechelen E, Vanderstichele H, Davidsson P, et al. Quantification of tau phosphorylated at threonine 181 in human cerebrospinal fluid: a sandwich ELISA with a synthetic phosphopeptide for standardization. Neurosci Lett. 2000;285:49-52.

5. Sunderland T, Linker G, Mirza N, et al. Decreased beta- amyloid 1-42 and increased tau levels in cerebrospinal fluid of patients with Alzheimer's disease. JAMA. 2003;289:2094-103.

6. Tapiola T, Alafuzoff I, Herukka SK, et al. Cerebrospinal fluid [beta]amyloid 42 and tau proteins as biomarker changes in the brain. Arch Neurol. 2009;66:382-9.

7. Shaw LM, Vanderstichele H, Knapik-Czajka M, Alzheimer's Disease Neuroimaging Initiative, et al. Cerebrospinal fluid biomarker signature in Alzheimer's disease neuroimaging initiative subjects. Ann Neurol. 2009;65:403-13.

8. Visser PJ, Verhey F, Knol DL, et al. Prevalence and prognostic value of cerebrospinal fluid markers of Alzheimer pathology in subjects with subjective cognitive impairment and mild cognitive impairment. The DeSCRIPA study. Lancet Neurol. 2009;8:619-27.

9. Mattsson N, Zetterberg H, Hansson O, et al. CSF bio- markers and incipient Alzheimer disease in patients with mild cognitive impairment. JAMA. 2009;302:385-93.

10. Jack CR Jr, Albert MS, Knopman DS, et al. Introduction to the recommendations from the National Institute on Aging-Alzheimer's Association workgroups on diagnostic guidelines for Alzheimer's disease. Alzheimers Dement. 2011;7(3):257-62.

11. McKhann GM, Knopman DS, Chertkow H, et al. The diagnosis of dementia due to Alzheimer's disease: recommendations from the National Institute on Aging-Alzheimer's Association workgroups on diagnostic guidelines for Alzheimer's disease. Alzheimers Dement. 2011;7(3):263-9.

12. Albert MS, DeKosky ST, Dickson D, et al. The diagnosis of mild cognitive impairment due to Alzheimer's disease: recommendations from the 
National Institute on Aging-Alzheimer's Association workgroups on diagnostic guidelines for Alzheimer's disease. Alzheimers Dement. 2011;7(3):270-9.

\section{Sperling RA, Aisen PS, Beckett LA, et al. Toward defining the} preclinical stages of Alzheimer's disease: recommendations from the National Institute on Aging-Alzheimer's Association workgroups on diagnostic guidelines for Alzheimer's disease. Alzheimers Dement. 2011;7(3):280-92.

14. Alzforum. Alzbiomarker [web-based resource]. Alzheimer's disease vs control: neurogranin (CSF). http://www.alzforum.org/alzbiomarker/metaanalysis/alzheimers-disease-vs-control-neurogranin-csf. Accessed 30 May 2016.

15. Alzforum. Alzbiomarker [web-based resource]. MCI-AD vs MCI-stable: neurogranin (CSF). http://www.alzforum.org/alzbiomarker/metaanalysis/mci-ad-vs-mci-stable-neurogranin-csf. Accessed 30 May 2016.

16. Alzforum. Alzbiomarker [web-based resource]. Alzheimer's disease vs control: hFABP (CSF). http://www.alzforum.org/alzbiomarker/metaanalysis/alzheimers-disease-vs-control-hfabp-csf. Accessed 30 May 2016.

17. Alzforum. Alzbiomarker [web-based resource]. Alzheimer's disease vs control: NSE (CSF). http://www.alzforum.org/alzbiomarker/metaanalysis/alzheimers-disease-vs-control-nse-csf. Accessed 30 May 2016.

18. Alzforum. Alzbiomarker [web-based resource]. Alzheimer's disease vs control: NFL (CSF). http://www.alzforum.org/alzbiomarker/metaanalysis/alzheimers-disease-vs-control-nfl-csf. Accessed 30 May 2016.

19. Alzforum. Alzbiomarker [web-based resource]. Alzheimer's disease vs control: YKL-40 (CSF)]. http://www.alzforum.org/alzbiomarker/metaanalysis/alzheimers-disease-vs-control-ykl-40-csf. Accessed 30 May 2016.

20. Alzforum. Alzbiomarker [web-based resource]. Alzheimer's disease vs control: VLP-1 (CSF). http://www.alzforum.org/alzbiomarker/metaanalysis/alzheimers-disease-vs-control-vlp-1-csf. Accessed 30 May 2016. 
21. Wellington H, Paterson RW, Portelius E, et al. Increased CSF neurogranin concentration is specific to Alzheimer disease. Neurology. 2016;86(9):829-35.

22. Paterson RW, Toombs J, Slattery CF, et al. Dissecting IWG-2 typical and atypical Alzheimer's disease: insights from cerebrospinal fluid analysis. J Neurol. 2015;262(12):2722-30.

23. Kroksveen AC, Opsahl JA, Aye TT, et al. Proteomics of human cerebrospinal fluid: discovery and verification of biomarker candidates in neurodegenerative diseases using quantitative proteomics. J Proteomics. 2011;74(4):371-88.

24. Wildsmith KR, Schauer SP, Smith AM, et al. Identification of longitudinally dynamic biomarkers in Alzheimer's disease cerebrospinal fluid by targeted proteomics. Mol Neurodegener. 2014;9:22.

25. Toledo JB, Vanderstichele H, Figurski M, et al. Alzheimer's disease neuroimaging initiative: factors affecting Abeta plasma levels and their utility as biomarkers in ADNI. Acta Neuropathol. 2011;122:401-13.

26. Huang Y, Potter R, Sigurdson W, et al. Beta-amyloid dynamics in human plasma. Arch Neurol. 2012;69:1591-7.

27. Le Bastard N, Aerts L, Leurs J, et al. No correlation between timelinked plasma and CSF Abeta levels. Neurochem Int. 2009;55:820-825.

28. Hansson O, Zetterberg H, Vanmechelen E, et al. Evaluation of plasma Abeta[40] and Abeta[42] as predictors of conversion to Alzheimer's disease in patients with mild cognitive impairment. Neurobiol Aging. 2010;31:35767.

29. Devanand DP, Schupf N, Stern Y, et al. Plasma Abeta and PET PiB binding are inversely related in mild cognitive impairment. Neurology. 2011;77:125-131.

30. Lui JK, Laws SM, Li QX, AIBL Research Group, et al. Plasma amyloidbeta as a biomarker in Alzheimer's disease: the AIBL study of aging. J Alzheimers Dis. 2010;20:1233-42. 
31. Figurski MJ, Waligorska T, Toledo J, et al. Improved protocol for measurement of plasma beta-amyloid in longitudinal evaluation of Alzheimer's Disease Neuroimaging Initiative study patients. Alzheimers Dement. 2012;8:250-60.

32. Olsson B, Lautner R, Andreasson U, et al. CSF and blood biomarkers for the diagnosis of Alzheimer's disease: a systematic review and metaanalysis. Lancet Neurol. 2016;15(7):673-84.

33. Alzforum. Alzbiomarker [web-based resource]. http://www.alzforum.org/alzbiomarker. Accessed 9 May 2016.

34. Sparks DL, Kryscio RJ, Sabbagh MN, et al. Tau is reduced in AD plasma and validation of employed ELISA methods. Am J Neurodegener Dis. 2012;1(1):99-106.

35. Wang T, Xiao S, Liu Y, et al. The efficacy of plasma biomarkers in early diagnosis of Alzheimer's disease. Int J Geriatr Psychiatry. 2014;29(7):713-9.

36. Chiu MJ, Yang SY, Horng HE, et al. Combined plasma biomarkers for diagnosing mild cognition impairment and Alzheimer's disease. ACS Chem Neurosci. 2013;4(12):1530-6.

37. Chiu MJ, Chen YF, Chen TF, et al. Plasma tau as a window to the brainnegative associations with brain volume and memory function in mild cognitive impairment and early Alzheimer's disease. Hum Brain Mapp. 2014;35(7):3132-42.

38. Zetterberg H, Wilson D, Andreasson U, et al. Plasma tau levels in Alzheimer's disease. Alzheimers Res Ther. 2013;5(2):9.

39. Wilson DH, Rissin DM, Kan CW, et al. The Simoa HD-1 analyzer: a novel fully automated digital immunoassay analyzer with single-molecule sensitivity and multiplexing. J Lab Autom. 2016;21(4):533-47.

40. Janelidze S, Stomrud E, Palmqvist $S$ et al. Plasma $\beta$-amyloid in Alzheimer's disease and vascular disease. Scientific Reports 2016; 6: 26801. doi:10.1038/srep26801 [Epub ahead of print].

\section{AQ11}


41. Dage JL, Wennberg AMV, Airey DC, et al. Levels of tau protein in plasma are associated with neurodegeneration and cognitive function in a population based elderly cohort. Neurology. 2016 pii: S1552-

5260(16)30087-5. doi: 10.1016/j.jalz.2016.06.001. [Epub ahead of print]

42. Neselius S, Zetterberg H, Blennow K, et al. Olympic boxing is associated with elevated levels of the neuronal protein tau in plasma. Brain Inj. 2013;27(4):425-33.

43. de Jong D, Jansen RW, Pijnenburg YA, et al. CSF neurofilament proteins in the differential diagnosis of dementia. J Neurol Neurosurg Psychiatry. 2007;78(9):936-8.

44. Scherling CS, Hall T, Berisha F, et al. Cerebrospinal fluid neurofilament concentration reflects disease severity in frontotemporal degeneration. Ann Neurol. 2014;75(1):116-26.

45. Wilke C, Preische O, Deuschle C, et al. Neurofilament light chain in FTD is elevated not only in cerebrospinal fluid, but also in serum. J Neurol Neurosurg Psychiatry. 2016. doi:10.1136/jnnp-2015-312972 (Epub 2016 May 17).

46. Rohrer JD, Woollacott IOC, Dick KM, et al. Serum neurofilament light chain protein is a measure of disease intensity in frontotemporal dementia. Neurology. 2016, 87(13):1329-36

47. Bacioglu M, Maia LF, Preische O, et al. Neurofilament light chain in blood and CSF as marker of disease progression in mouse models and in neurodegenerative diseases. Neuron. 2016;91:1-11.

48. Kuhle J, Barro C, Andreasson U, et al. Comparison of three analytical platforms for quantification of the neurofilament light chain in blood samples: ELISA, electrochemiluminescence immunoassay and Simoa. Clin Chem Lab Med. 2016;54(10):1655-61. doi:10.1515/cclm-2015-1195. 
neurofilament light protein (NFL) is a biomarker of CNS injury in HIV infection: a cross-sectional study. EBioMedicine. 2015;22(3):135-40.

50. O'Bryant SE, Gupta V, Henriksen K, STAR-B and BBBIG working groups, et al. Guidelines for the standardization of preanalytic variables for blood-based biomarker studies in Alzheimer's disease research. Alzheimers Dement. 2015;11(5):549-60.

51. Ray S, Britschgi M, Herbert C, et al. Classification and prediction of clinical Alzheimer's diagnosis based on plasma signaling proteins. Nat Med. 2007;13(11):1359-62.

52. Soares HD, Chen Y, Sabbagh M, et al. Identifying early markers of Alzheimer's disease using quantitative multiplex proteomic immunoassay panels. Ann N Y Acad Sci. 2009;1180:56-67.

53. Björkqvist M, Ohlsson M, Minthon L, et al. Evaluation of a previously suggested plasma biomarker panel to identify Alzheimer's disease. PLoS One. 2012;7(1):e29868.

54. Casanova R, Varma S, Simpson B, et al. Blood metabolite markers of preclinical Alzheimer's disease in two longitudinally followed cohorts of older individuals. Alzheimers Dement. 2016;12(7):815-22.

55. Doecke JD, Laws SM, Faux NG, et al. Blood-based protein biomarkers for diagnosis of Alzheimer disease. Arch Neurol. 2012;69(10):1318-1325.

56. Zhang R, Barker L, Pinchev D, et al. Mining biomarkers in human sera using proteomic tools. Proteomics. 2004;4(1):244-56.

57. Trushina E, Dutta T, Persson XT, et al. Identification of altered metabolic pathways in plasma and CSF in mild cognitive impairment and Alzheimer's disease using metabolomics. PLoS One. 2013;8(5):e63644.

58. Sattlecker M, Kiddle SJ, Newhouse S, et al. AddNeuroMed Consortium. Alzheimer's disease biomarker discovery using SOMAscan multiplexed protein technology. Alzheimers Dement. 2014;10(6):724-34. 
proteomic profiling of Alzheimer disease samples reveals disease-specific, carrier-protein-bound mass signatures. Clin Chem. 2005;51:1946-54.

60. Jaeger PA, Lucin KM, Britschgi M, et al. Network-driven plasma proteomics expose molecular changes in the Alzheimer's brain. Mol Neurodegener. 2016;11:31. doi:10.1186/s13024-016-0095-2.

61. Hu WT, Holtzman DM, Fagan AM, et al. Plasma multianalyte profiling in mild cognitive impairment and Alzheimer disease. Neurology. 2012;79:897-905.

62. Mapstone M, Cheema AK, Fiandaca MS, et al. Plasma phospholipids identify antecedent memory impairment in older adults. Nat Med. 2014;20:415-8.

63 Heywood WE, Galimberti D, Bliss E et al. Identification of novel CSF biomarkers for neurodegeneration and their validation by a high-throughput multiplexed targeted proteomic assay. Molecular Neurodegeneration $2015 ; 10: 64$.

64 Heywood W, Galimberti D, Bliss E et al. 